\title{
Effect of Alpha-Linolenic Acid on Oocyte Maturation and Embryo Development in Pigs
}

\author{
Ji-Eun Lee ${ }^{1}$, Yong Hwangbo ${ }^{1}$, Hwa-Young Kim¹, Won-Hee Lee ${ }^{1}$, Hee-Tae Cheong ${ }^{2}$, \\ Boo-Keun Yang ${ }^{1}$ and ${ }^{\dagger}$ Choon-Keun Park ${ }^{1}$ \\ ${ }^{1}$ College of Animal Life Sciences, Kangwon National University, Chunchoen 24341, Republic of Korea \\ ${ }^{2}$ College of Veterinary Medicine, Kangwon National University, Chunchoen 24341, Republic of Korea
}

\begin{abstract}
The aim of this study was to determine the effect of additional alpha-linolenic acid (ALA) supplementation during in vitro maturation (IVM) and culture (IVC) on nucleic maturation and embryo development of pigs. Cumulus-oocyte complexes (COCs) were incubated in IVM medium containing different concentration of ALA (25, 50 and $100 \mu \mathrm{M})$ for $44 \mathrm{~h}$. After in vitro maturation, nuclear maturation of oocytes were evaluated by aceto-orcein stain. Mature oocytes with $50 \mu \mathrm{M}$ ALA were fertilized and cultured in IVC medium with ALA (25, 50 and $100 \mu \mathrm{M})$ during early-embryogenesis (48 hours after fertilization). Then, embryos were cultured with $25 \mu \mathrm{M}$ ALA during early embryogenesis and/or late embryogenesis (120 hours after early-embryogenesis). In results, oocyte maturation were significantly increased by $50 \mu \mathrm{M}$ ALA treatment groups compared with control groups $(p<0.05)$. Treatment of $25 \mu \mathrm{M}$ ALA during early-embryogenesis enhanced cleavage rate of embryo compared with other groups $(p<0.05)$, whereas formation and total cell number of blastocyst had no significant difference. Similarly, cleavage rate of embryos were increased by $25 \mu \mathrm{M}$ ALA supplement during early- or late-embryogenesis than ALA treatment both stage of embryogenesis $(p<0.05)$, but did not influence to blastocyst formation. Interestingly, total cell number of blastocyst were enhanced in ALA treatment group during early-embryogenesis. These findings indicated that ALA supplement enhance the nuclear maturation of oocyte and embryo development, however, excessive ALA could negatively influence. Therefore, we suggest that ALA is used for improvement of in vitro production of mammalian embryo and further study regarding with functional mechanism of ALA is needed.
\end{abstract}

Key words : Alpha-linolenic acid, In vitro maturation, In vitro culture, Oocyte, Embryo, Pig

\section{INTRODUCTION}

Polyunsaturated fatty acids (PUFAs) are one of fatty acid that have two or more double bonds and exist as cisor trans-isoform following by structure of double bonds. The follicles, oocytes, and spermatozoa contain large amount of PUFAs (Homa \& Brown, 1992). Especially, biochemical composition of follicular fluid determines microenvironment in follicles for oocyte growth and maturation (Khalil et al., 2013) and PUFAs influence to oocyte maturation, fertilization, and subsequent development through altering the biochemical composition of follicular fluid (Bilby et al., 2006). Fouladi-Nashta et al. (2009) had reported that fatty acid composition in granulosa cells were altered by dietary or supplementation in culture medium, and it had influence to oocyte maturation, and subsequent

\footnotetext{
Manuscript received May 27, 2017, Received in revised form May 31, 2017, Accepted June 2, 2017

$\dagger$ Corresponding Author : Choon-Keun Park, College of Animal Life Sciences, Kangwon National University, Chuncheon 24341, Republic of Korea, Tel : +82-33-250-8627, E-mail : parkck@kangwon.ac.kr

This is an Open Access article distributed under the terms of the Creative Commons Attribution Non-Commercial License (http:// creativecommons.org/licenses/by-nc/3.0) which permits unrestricted non-commercial use, distribution, and reproduction in any medium, provided the original work is properly cited.
} 
development in in vivo and in vitro. Omega-3, a one of PUFAs, regulates variety of reproductive function in mammals (Wathes et al., 2007). Quality of oocyte and embryo were enhanced by their type and ratio in composition of fatty acid (Wonnacott et al., 2010).

Alpha-linolenic acid (ALA; n-3 18:3), a one of omega-3 fatty acid, is mainly contained in chloroplast of plant (Marei et al., 2009). As an essential fatty acid, ALA was not synthesized in mammalian body including human, cow, and pig. And it must be supplied from foods (Sturmey et al., 2009). Ambrose et al. (2006) had reported that dietary ALA in cows increased the size of pre-ovulatory follicles and reduced pregnancy loss. Also, supplementation of ALA during bovine oocyte maturation decreased intracellular reactive oxygen species (ROS) level (Marei et al., 2012). Many studies that are involved with effect of ALA by dietary or in vitro supplement on female reproductive events in cows were reported. However, a few studies in pigs were reported.

Pigs are important animal not only for agriculture, but also for research of human disease as a disease model animal (Pratt et al., 2006). Because pigs have genetic, anatomical, and physiological similarity compared to human, it was used in various field of research for disease model and production of transgenic animals (Lin et al., 2009). Successful in vitro production (IVP) technique of porcine embryos were required for various researches, however, quality of embryo derived from IVP in pigs was lower than embryo derived from in vivo (Kitagawa et al., 2004). In in vitro environment, porcine oocytes have a lower maturation rate in both of nuclear and cytoplasm than in vivo mature oocytes (Kim et al., 2013; Elahi et al., 2016). To improve these problems, many researchers supplied the antioxidants and fatty acid into maturation or culture medium of oocytes, and fatty acids play a role as an energy source of oocytes, storage of energy for metabolism, and embryo development (McKeegan \& Sturmey, 2011). Based on these studies, we expected that function of ALA positively influenced to oocyte maturation and subsequent development. Therefore, the aim of this study was to investigate the effect of additional ALA during in vitro maturation (IVM) and culture (IVC) on nuclear maturation and embryo development in pigs.

\section{MATERIALS AND METHODS}

\section{In vitro maturation (IVM)}

All procedures that involved the use of animals were approved by the Kangwon National University Institutional Animal Care and Use Committee (KIACUC-09-0139). Ovaries were collected from slaughtered gilts at local slaughter house and transferred into the laboratory in $0.9 \%$ (w/v) sterilized saline within $2 \mathrm{~h}$. The cumulus-oocyte complexes (COCs) were aspirated from antral follicles with 3-6 $\mathrm{mm}$ in diameter using $10 \mathrm{cc}$ syringe with 18-gauge needle. After aspiration, COCs with homogeneous cytoplasm and compact cumulus layer were selected and incubated in medium-199 (Invitrogen, MA, USA) containing $10 \%(\mathrm{v} / \mathrm{v})$ porcine follicular fluid $(\mathrm{pFF}), 10 \mathrm{IU} / \mathrm{mL}$ human chorionic gonadotropin (hCG; Intervet), $10 \mathrm{ng} / \mathrm{mL}$ luteinizing hormone (LH; Sigma-Aldrich, St. Louis, MO, USA), $10 \mathrm{ng} / \mathrm{mL}$ follicle stimulating hormone (FSH; Sigma-Aldrich) and $10 \mathrm{ng} / \mathrm{mL}$ epidermal growth factor (EGF; Sigma-Aldrich) with ALA, which was diluted using ethanol (final concentration: $0.9 \%$ ), at $38.5^{\circ} \mathrm{C}, 5 \% \mathrm{CO}_{2}$ condition for $22 \mathrm{~h}$. Because hCG is known that it can improve cytoplasmic maturation (Kątska-Książkiewicz, 2006), we added hCG into IVM medium. Then, they were subsequently incubated using hormone-free medium-199 with ALA at $38.5^{\circ} \mathrm{C}, 5 \% \mathrm{CO}_{2}$ condition for $22 \mathrm{~h}$.

\section{Evaluation of nuclear maturation}

Aceto-orcein stain method was used to evaluate nuclear maturation stage of porcine oocytes that were incubated with different concentration of ALA during IVM. Hyaluronidase was treated into mature COCs and cumulus cells 
were removed by gentle pipetting using pasture pipette. Denuded oocytes were fixed in acetic alcohol solution (acetic acid:ethanol; $1: 3$; v/v) for $48 \mathrm{~h}$, at room temperature (RT). And oocytes were was stained by $1 \%(\mathrm{w} / \mathrm{v})$ aceto-orcein at RT for $7 \mathrm{~min}$. The morphology of nuclear was observed under light microscope. Metaphase II (MII) stage oocytes were classified as mature oocyte.

\section{In vitro fertilization (IVF)}

Mature COCs were treated by $0.1 \%(\mathrm{v} / \mathrm{v})$ hyaluronidase for reduction of binding between cumulus cells. And they placed in $50 \mu \mathrm{L}$ drop of modified tris-buffered medium (mTBM) containing $0.2 \%(\mathrm{w} / \mathrm{v})$ bovine serum albumin (BSA; Sigma-Aldrich). For hyperactivation of spermatozoa, fresh boar semen were placed in mTBM containing $0.4 \%(\mathrm{w} / \mathrm{v})$ BSA and $0.39 \mathrm{mg} / \mathrm{mL}$ caffeine (Sigma-Aldrich) and they diluted at a final concentration of $6 \times 10^{5}$ spermatozoa $/ \mathrm{mL}$. Activated sperm inseminated into prepared COCs and co-incubated at $38.5^{\circ} \mathrm{C}, 5 \% \mathrm{CO}_{2}$ condition for $6 \mathrm{~h}$.

\section{In vitro culture (IVC)}

After fertilization, cumulus cells and spermatozoa surrounding zona pellucida of fertilized oocytes were completely removed by gentle pipetting and 70-80 embryos were cultured in 4-well dishes (SPL Life Science, Korea) with $650 \mu \mathrm{L}$ porcine zygote medium-3 (PZM-3) containing $0.3 \%(\mathrm{w} / \mathrm{v}) \mathrm{BSA}$ (Sigma-aldrich) at $38.5^{\circ} \mathrm{C}, 5 \% \mathrm{CO}_{2}$ condition for $48 \mathrm{~h}$ (early-embryogenesis). Then, culture medium was exchanged to fresh medium and subsequently incubated for $120 \mathrm{~h}$ in same culture condition (late-embryogenesis). For comparison of embryo development between short- and long-term ALA treatment at $168 \mathrm{~h}$ after IVF, cleavage rate, blastocyst formation, and total cell number of blastocyst were checked at $168 \mathrm{~h}$ after IVF.

\section{Total cell number of blastocyst}

To evaluate total cell number, blastocyst stage embryos were fixed in $4 \%(\mathrm{v} / \mathrm{v})$ paraformaldehyde at room tempera- ture (RT) for $3 \mathrm{~min}$. And nuclear of blastocyst was stained by $1 \mu \mathrm{g} / \mathrm{mL}$ bisbenzimide H 33342 trihydrochloride (Hoechst 33342; Sigma-Aldrich) at RT for $30 \mathrm{~min}$ in dark room and observed using fluorescent microscope.

\section{Statistical analysis}

All numerical data representing each parameter were analyzed using the Statistical Analysis System Software (SAS, version 9.4). Data were presented means \pm SEM and comparisons among treatment groups were conducted by least significant difference (LSD) tests using a generalized linear model (GLM) in the SAS package. A value of $p<$ 0.05 was considered to indicate a statistically significant difference.

\section{Experimental designs}

\section{1) Experiment 1: Effect of ALA on nuclear matura-} tion of porcine oocytes

To evaluate effect of ALA on nuclear maturation of oocytes, porcine oocytes were incubated using IVM medium with different concentration of ALA $(0,25,50$, and 100 $\mu \mathrm{M}$; Sigma-aldrich) for $44 \mathrm{~h}$ and stage of nuclear maturation was observed using aceto-orcein stain method. In experiment 1, 50 COCs were used in each replication and total 3 replication were conducted.

\section{2) Experiment 2: Effect of ALA during early-embryo-} genesis on embryo development

Based on result in experiment 1 , mature oocytes with 50 $\mu \mathrm{M}$ ALA were fertilized and cultured using IVC medium containing different concentration of $\operatorname{ALA}(0,25,50$, and $100 \mu \mathrm{M}$ ) for $48 \mathrm{~h}$ after fertilization (early-embryogenesis). Then, culture medium without ALA was exchanged and embryos were subsequently cultured for $120 \mathrm{~h}$.

\section{3) Experiment 3: Effect of ALA during early- and/or} late-embryogenesis on embryo development 
In pigs, the early embryos develop in oviduct and they are transported into the uterus at $48 \mathrm{~h}$ after fertilization. Therefore, we expected that embryo development was differently influenced before or after at $48 \mathrm{~h}$ after fertilization. To investigate the effect of ALA during embryogenesis, mature embryos with ALA were cultured in IVC medium with or without $25 \mu \mathrm{M}$ ALA for $48 \mathrm{~h}$ and subsequently incubated using IVC medium with or without $25 \mu \mathrm{M}$ ALA for $120 \mathrm{~h}$. Treatment groups were named as following: T1, without ALA in early- and late-embryogenesis; T2, with ALA in late-embryo-genesis; T3, with ALA in early-embryogenesis; T4, with ALA in early- and late-embryogenesis.

\section{RESULTS}

1. Effect of ALA on nuclear maturation of porcine oocytes

The Effect of ALA during IVM on nuclear maturation of porcine oocytes was shown in Table 1. Mature oocytes, which are reached at MII stage, were increased by $50 \mu \mathrm{M}$ ALA treatment $(P<0.05)$, whereas $25 \mu \mathrm{M}$ ALA did not influence. Treatment of $100 \mu \mathrm{M}$ ALA reduced oocyte maturation compared to $50 \mu \mathrm{M}$ ALA groups, however, there is no significant difference.

2. Effect of ALA during early-embryogenesis on embryo development

Table 2 indicated the embryo development by different concentration of ALA during early-embryogenesis. The

Table 1. Effects of alpha-linolenic acid on nuclear maturation during in vitro maturation in porcine oocytes.

\begin{tabular}{cccc}
\hline \hline \multirow{2}{*}{ ALA $(\mu \mathrm{M})$} & $\begin{array}{c}\text { No. of oocytes used } \\
\text { for IVM }(\%)\end{array}$ & \multicolumn{2}{c}{ No. of oocyte $(\%)$} \\
\cline { 3 - 4 } & 221 & $26(17.3 \pm 1.8)^{\mathrm{a}}$ & Mature \\
\hline 0 & 226 & $26(17.3 \pm 1.3)^{\mathrm{a}}$ & $124(82.7 \pm 1.7)^{\mathrm{b}}$ \\
25 & 229 & $16(10.7 \pm 1.8)^{\mathrm{b}}$ & $124(82.7 \pm 1.3)^{\mathrm{b}}$ \\
50 & 226 & $23(15.3 \pm 1.8)^{\mathrm{ab}}$ & $134(89.3 \pm 1.8)^{\mathrm{a}}$ \\
100 & & Immature & $127(85.3 \pm 1.8)^{\mathrm{ab}}$ \\
\hline
\end{tabular}

${ }^{\mathrm{a}, \mathrm{b}}$ Mean values with different superscripts are significantly difference $(P<0.05)$.

Data are presented as mean $\pm \mathrm{SEM}$.

Table 2. Effects of alpha-linolenic acid on embryo development of mature oocytes with alpha-linolenic acid in pigs

\begin{tabular}{cccc}
\hline \multirow{2}{*}{ ALA $(\mu \mathrm{M})$} & $\begin{array}{c}\text { No. of oocytes used } \\
\text { for IVF }(\%)\end{array}$ & \multicolumn{2}{c}{ No. of embryo development to (\%) } \\
\cline { 2 - 4 } & 221 & $178(80.5 \pm 0.1)^{\mathrm{b}}$ & Blastocyst \\
\hline 0 & 226 & $199(88.1 \pm 0.1)^{\mathrm{a}}$ & $15(10.4 \pm 1.3)$ \\
25 & 229 & $180(78.6 \pm 0.3)^{\mathrm{bc}}$ & $20(12.1 \pm 2.1)$ \\
50 & 226 & $173(76.5 \pm 1.7)^{\mathrm{c}}$ & $14(8.9 \pm 0.4)$ \\
\hline
\end{tabular}

${ }^{a, b}$ Mean values with different superscripts are significantly difference $(P<0.05)$.

Oocytes were maturated with $50 \mu \mathrm{M}$ ALA for $44 \mathrm{~h}$.

Data are presented as mean \pm SEM. 
cleavage rate in $25 \mu \mathrm{M}$ ALA-treated groups were significantly higher than other treatment groups $(P<0.05)$. On the other hand, treatment of 50 and $100 \mu \mathrm{M}$ ALA reduced cleavage rates compared to control group, especially, it was significantly decreased in $100 \mu \mathrm{M}$ ALA group compared with control and $25 \mu \mathrm{M}$ ALA groups $(P<0.05)$. Beside, blastocyst formation and total cell number were not influenced by ALA treatment (Fig. 1).

3. Effect of ALA during early- and/or late-embryogenesis on embryo development on embryo development

The effect of long- and/or short-supplement of ALA on embryo development were presented in Table 3 and Fig. 2. $\mathrm{T} 2$ and $\mathrm{T} 3$ treatment increased cleavage rate compared to $\mathrm{T} 1$, however, there was no significant difference. And clea vage rate in T4 group was significantly lower than T2 groups $(P<0.05)$, whereas it was no difference with control and T2 groups. Similar to result in experiment 2, ALA treatment did not influence to blastocyst formation. And blastocysts in $\mathrm{T} 4$ treatment group had lower number of cells than T3 group $(P<0.05)$.
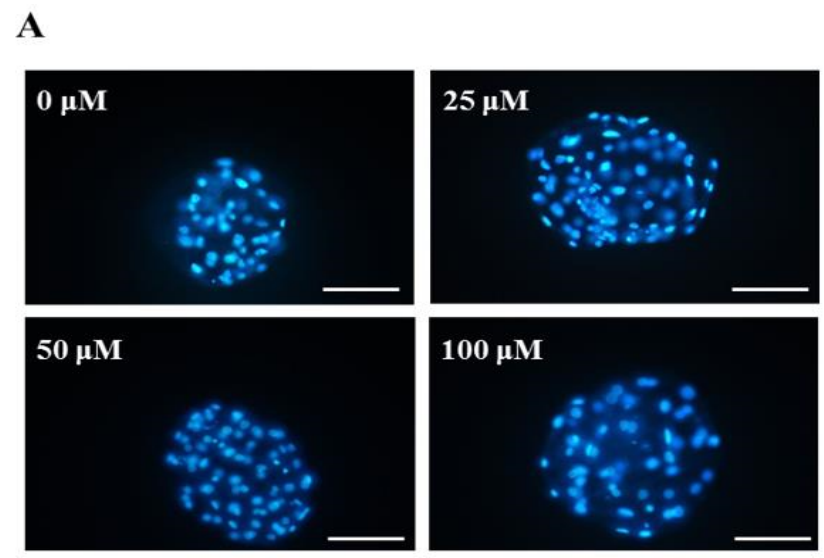

B

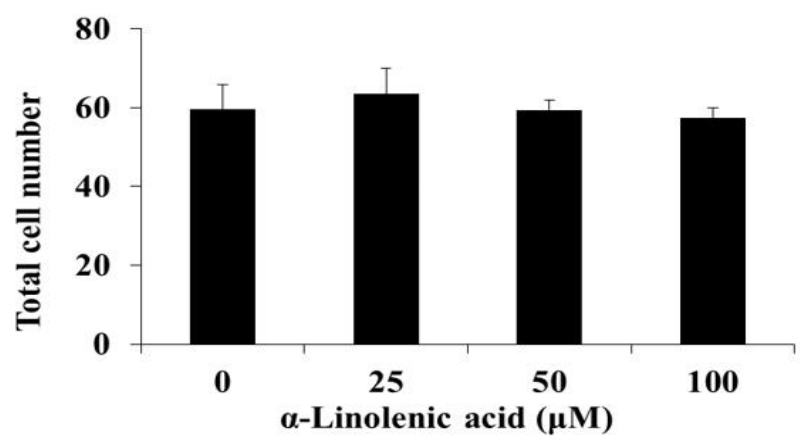

Fig. 1. Effect of alpha-linolenic acid during early-embryogenesis on total cell number of porcine blastocysts at $168 \mathrm{~h}$ after in vitro fertilization. (A) fluorescence image of stained nuclear by Hoechst 33342, (B) total cell number of blastocyst. Scale bar: $200 \mu \mathrm{m}$.

Table 3. Effects of alpha-linolenic acid on early- and/or late-embryogenesis of maturd oocyte with alpha-linolenic acid in pigs

\begin{tabular}{cccc}
\hline \hline \multirow{2}{*}{$\begin{array}{c}\text { Treatment } \\
\text { groups }\end{array}$} & $\begin{array}{c}\text { No. of oocytes used } \\
\text { for IVF }(\%)\end{array}$ & Cleavage & Blastocyst \\
\cline { 3 - 4 } T 1 & 150 & $132(88.0 \pm 3.1)^{\mathrm{ab}}$ & $18(12.0 \pm 3.1)$ \\
T 2 & 150 & $139(92.7 \pm 0.7)^{\mathrm{a}}$ & $15(10.0 \pm 2.3)$ \\
T 3 & 150 & $141(94.0 \pm 1.2)^{\mathrm{a}}$ & $16(10.7 \pm 2.4)$ \\
T 4 & 150 & $125(83.3 \pm 1.8)^{\mathrm{b}}$ & $8(5.3 \pm 1.3)$ \\
\hline
\end{tabular}

${ }^{a, b}$ Mean values with different superscripts within a column are significantly difference $(P<0.05)$.

Oocytes were maturated with $50 \mu \mathrm{M}$ ALA for $44 \mathrm{~h}$.

T1: without ALA both early- and late-embryogenesis; T2: with ALA at late embryogenesis; T3: with ALA at early embryogenesis; T4: with ALA for both early- and late-embryogenesis. 
A
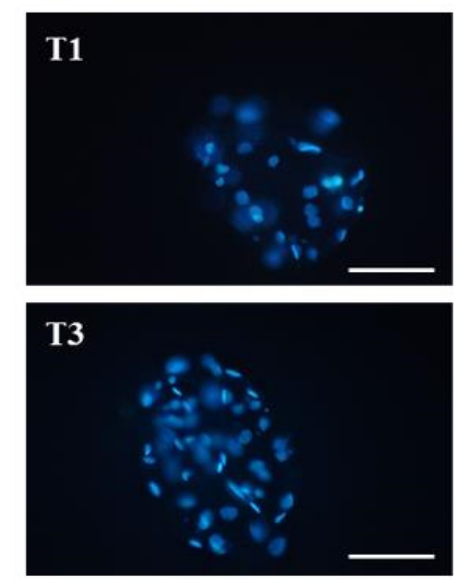

B

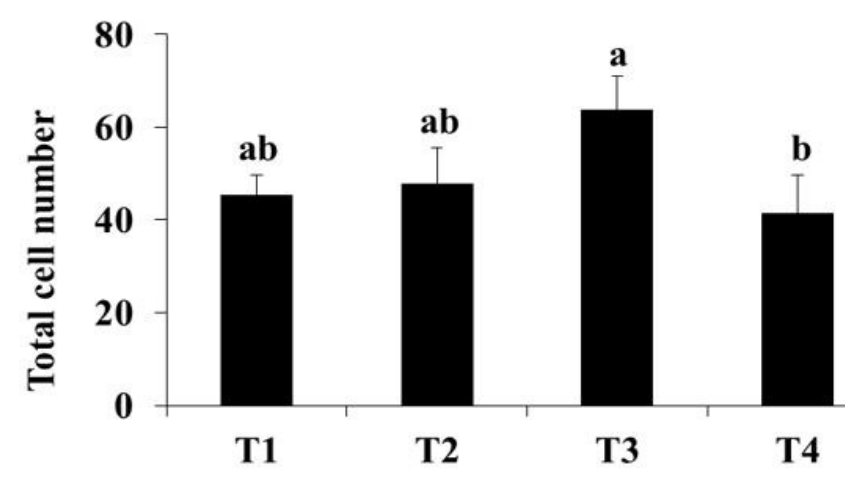

Fig. 2. Effect of alpha-linolenic acid during early- and/ or late-embryogenesis on total cell number of porcine blastocysts at $168 \mathrm{~h}$ after in vitro fertilization. (A): fluorescence image of stained nuclear by Hoechst 33342, (B): total cell number of blastocyst. T1: without ALA both early- and late-embryogenesis; T2; with $25 \mu \mathrm{M}$ ALA at late embryogenesis, T3; with $25 \mu \mathrm{M}$ ALA at early embryogenesis, T4; with $25 \mu \mathrm{M}$ ALA for both early- and late-embryogenesis Scale bar: $200 \mu \mathrm{m}$. $(P<0.05)$.

\section{DISCUSSION}

Many researchers used $\mathrm{pFF}$ for improvement of porcine IVP system through imitation of in vivo environment. Oocyte maturation in in vivo environment was occurred in follicles that contain follicular fluids and influenced by nutrients in follicular fluid. And composition of follicular fluid are changed by dietary nutrients. Therefore, this study was conducted to investigate effect of additional ALA on nuclear maturation and embryo development in pigs. Our findings showed that nuclear maturation of porcine oocytes were enhanced by $50 \mu \mathrm{M}$ ALA treatment, whereas it was reduced by treatment of $100 \mu \mathrm{M}$ ALA. In addition, supplement of $25 \mu \mathrm{M}$ ALA during early-embryogenesis increased cleavage rate of embryo that were mature with 50 $\mu \mathrm{M}$ ALA, however, blastocyst formation and total cell number were not influenced by treatment of ALA. Interestingly, $25 \mu \mathrm{M}$ ALA treatment during early-embryogenesis enhanced cleavage rate and blastocyst formation, on the contrary, ALA treatment during both of early- and lateembryogenesis decreased embryo development and quality.

Fatty acid is considered as potential metabolic substrate in oocyte and early stage embryo and required as energy source for metabolism in embryo during pre-implantation in pigs and cows (Sturmey et al., 2009). Wakefield et al. (2007) had reported that maternal supply of omega-3 PUFA influence to mature of oocyte through distribution of mitochondria, level of intracellular calcium, and increase of ROS during oocyte maturation in follicle. Also, cis-unsaturated fatty acid stimulates generation of ROS and lipid peroxidation in human spermatozoa (Aitken et al., 2006). These studies indicated that supplementation of fatty acids were associated with generation of ROS in various type of cells and it affected to cellular process such as oocyte maturation, embryo development, and survival via stimulation by ROS. ROS, which is produced by normal metabolism of the cell, is caused oxidative stress as a crucial signal molecule (Al-Gubory et al., 2010). Excessive ROS level in cells induce inactivation of enzymes, DNA fragmentation, dysfunction of mitochondria, and apoptosis (Kowaltowski \& vercesi, 1999).

Exposure of ROS reduces the oocyte maturation, fertilization and embryo development both in vivo and in vitro. In mouse oocytes, exposure of citrinin toxin during maturation increased ROS generation and apoptosis, and decreased maturation rates and blastocyst formation (Wu et 
al., 2017). In results of present study, nuclear maturation of porcine oocytes were enhanced by $50 \mu \mathrm{M}$ ALA treatment and these results were corresponded to research that was reported by Marei et al. (2009). However, Lee et al. (2016) had reported that nuclear maturation of porcine oocytes were enhanced by $100 \mu \mathrm{M}$ ALA that was diluted by culture media including $0.4 \% \mathrm{BSA}$, epidermal growth factor, and insulin. Thus, we expected that different result might be attributed to dilution method of ALA and composition of culture media used in each experiment. During the porcine oocyte maturation, intracellular glutathione (GSH) was increased by treatment of ALA (Lee et al., 2016) and Marei et al. (2012) had reported that ALA treatment reduced ROS level in bovine oocyte. GSH plays an important role in prevent of damage by oxidative stress (Nagai, 2001). Based on these results, our results indicated that ALA treatment during porcine oocyte maturation might enhance the nuclear maturation via reduction of oxidative stress. In addition, reduced nuclear maturation of porcine and bovine oocyte by mitogen-activated protein kinase (MAPK) inhibitor during in vitro maturation was recovered by ALA treatment (Marei et al., 2009; Lee et al., 2016) and these results suggested that ALA was involved with signal pathway regarding to nuclear maturation of oocytes.

Supplementation of $50 \mu \mathrm{M}$ ALA in IVM medium increased nuclear maturation of porcine oocytes, however, $100 \mu \mathrm{M}$ ALA treatment reduced oocyte maturation during IVM. Similar to our findings, 100 and $200 \mu \mathrm{M}$ ALA decreased nuclear maturation of bovine oocytes, whereas 50 $\mu \mathrm{M}$ ALA increased oocyte maturation (Marei et al., 2009). Wakefield et al. (2007) had reported that supply of omega3 fatty acid by dietary increased ROS level in murine oocytes and these results suggested that excessive ALA suppressed oocyte maturation by inducing of oxidative stress.

Measurement of $\mathrm{CO}_{2}$ in rabbit embryos when cultured with palmitic acid during the development of rabbit embryos shows that embryos can oxidize fatty acids even in the single-cell stage, In particular, it was reported that the production of $\mathrm{CO}_{2}$ increased from the 4 cell stage (Khandoker \& Tsujii,1998). This indicated that fatty acids added during embryonic development were used as an energy source (Sturmey et al., 2009), and thus the addition of 25 $\mu \mathrm{M}$ ALA during embryonic development in this study may have served as an energy source during embryonic development. However, treatment with $100 \mu \mathrm{M}$ ALA suggests that oxidative stress may be induced in the oocyte and inhibit development of the embryo.

Lee et al. (2016) had reported that $100 \mu \mathrm{M}$ ALA treatment during IVM of porcine oocytes enhanced embryonic development after parthenogenesis of oocytes. In present study, ALA was supplied into both of maturation and subsequent development process of porcine oocytes. Additional supplementation of $100 \mu \mathrm{M}$ ALA during IVC of porcine embryo negatively affected to cleavage rate of embryo after IVF. These different results suggested that embryo development of porcine oocytes were influenced by period of ALA treatment and fertilization method. Interestingly, total cell number of control group in experiment 3 was decreased compare with control group in experiment 2 . We expected that it was influenced by seasonal effect. Experiment 2 was conducted during winter, whereas experiment 3 was conducted during summer.

It is known that oxidative stress is a major obstacle to the development of embryos and that excessive ROS can negatively affect embryo development (Guerin et al., 2001). Antioxidants are added to reduce excessive ROS production during embryonic development, and antioxidants have been reported to be effective in embryonic development such as mice, pigs, and cows by lowering the ROS level in embryos (Takahashi, 2012). Lee et al. (2015) had reported that $200 \mu \mathrm{M} \gamma$-tocotrienol was reduced during embryonic development and decreased ROS and improved embryonic development. This is similar to the results of increased cleavage rates and total cell number in the T3 group treated with ALA during early embryonic development in this study. However, when $400 \mu \mathrm{M} \gamma$-tocotrienol 
was treated, the embryo apoptosis was increased as well as ROS in the embryo, This is similar to the results of the reduced cleavage rates and total cell number of blastocysts in the T4 group treated with ALA during both early and late embryonic development during after of fertilization development in this study, as a result, excessive ALA treatment had a negative effect on oocytes.

In this present study, supplementation of additional ALA in IVM and IVC medium enhanced nuclear maturation of oocyte and embryo development in pigs. However, high concentration of ALA (100 $\mu \mathrm{M}$ in IVM; 50 and $100 \mu \mathrm{M}$ in IVC) negatively affect to oocyte maturation and embryo development. Therefore, our findings suggest that ALA could improve in vitro production systems of porcine embryo and additional ALA in dietary may enhanced reproductive performance of pigs.

\section{ACKNOWLEDGEMENTS}

This work was supported by the National Research Foundation of Korea (NRF) grant funded by the Korea Government (Ministry of Education)(2016R1D1A1B03931746).

\section{REFERENCES}

Aitken RJ, Wingate JK, De Iuliis GN, Koppers AJ, McLaughlin EA (2006) Cis-unsaturated fatty acids stimulate reactive oxygen species generation and lipid peroxidation in human spermatozoa. J Clin Endocrinol Metab 91:4154-4163.

Al-Gubory KH, Fowler PA, Garrel C (2010) The role of cellular reactive oxygen species, oxidative stress and antioxidants in pregnancy outcomes. Int $\mathrm{J}$ Biochem Cell Biol 42:1634-1650.

Ambrose DJ, Kastelic JP, Corbett R, Pitney PA, Petit HV, Small JA, Zalkovic P (2006) Lower pregnancy losses in lactating dairy cows fed a diet enriched in alphalinolenic acid. J Dairy Sci 89:3066-3074.
Bilby TR, Block J, do Amaral BC, Sa Filho O, Silvestre FT, Hansen PJ, Staples CR, Thatcher WW (2006) Effects of dietary unsaturated fatty acids on oocyte quality and follicular development in lactating dairy cows in summer. J Dairy Sci 89:3891-3903.

Elahi F, Lee H, Lee Y, Park B, Lee J, Hyun SH, Lee E (2016) Cilostazol improves developmental competence of pig oocytes by increasing intraoocyte cyclic adenosine monophosphate level and delaying meiotic resumption. Reprod Domest Anim 51:220-226.

Fouladi-Nashta AA, Wonnacott KE, Gutierrez CG, Gong JG, Sinclair KD, Garnsworthy PC, Webb R (2009) Oocyte quality in lactating dairy cows fed on high levels of n-3 and n-6 fatty acids. Reproduction 138:771-781.

Guerin P, El Mouatassim S, Menezo Y (2001) Oxidative stress and protection against reactive oxygen species in the pre-implantation embryo and its surroundings. Hum Reprod 7:175-189.

Homa ST, Brown CA (1992) Changes in linoleic acid during follicular development and inhibition of spontaneous breakdown of germinal vesicles in cumulus-free bovine oocytes. J Reprod Fertil 94:153-160.

Kątska-Książkiewicz L (2006) Pig embryo production by in vitro maturation and fertilization of ovarian oocytes. A review. J Anim Feed Sci 15:525-542.

Khalil WA, Marei WF, Khalid M (2013) Protective effects of antioxidants on linoleic acid-treated bovine oocytes during maturation and subsequent embryo development. Theriogenology, 80:161-168.

Khandoker M, Tsujii H (1998). Metabolism of exogenous fatty acids by preimplantation rabbit embryos. Jpn J Fertil Steril 43:195-201.

Kim CH, Cheon YP, Lee YJ, Lee KH, Kim SH, Chae DH, Kang BM (2013) The effect of fibroblast co-culture on in vitro maturation of mouse preantral follicles. Dev Reprod 17:269-274.

Kitagawa Y, Suzuki K, Yoneda A, Watanabe T (2004) Effects of oxygen concentration and antioxidants on the 
in vitro developmental ability, production of reactive oxygen species (ROS), and DNA fragmentation in porcine embryos. Theriogenology 62:1186-1197.

Kowaltowski AJ, Vercesi AE (1999) Mitochondrial damage induced by conditions of oxidative stress. Free Radic Biol Med 26:463-71.

Lee S, Park EJ, Moon JH, Kim SJ, Song K, Lee BC (2015) Sequential treatment with resveratrol-trolox improves development of porcine embryos derived from parthenogenetic activation and somatic cell nuclear transfer. Theriogenology 84:145-154.

Lee Y, Lee H, Park B, Elahi J, Lee J, Lee ST, Park CK, Hyun SH, Lee E (2016) Alpha-linolenic acid treatment during oocyte maturation enhances embryonic development by influencing mitogen-activated protein kinase activity and intraoocyte glutathione content in pigs. J Anim Sci 94:3255-3263.

Lin L, Kragh PM, Purup S, Kuwayama M, Du Y, Zhang X, Yang H, Bolund L, Callesen H, Vajta G (2009) Osmotic stress induced by sodium chloride, sucrose or trehalose improves cryotolerance and developmental competence of porcine oocytes. Reprod Fertil Dev 21:338344.

Marei WF, Wathes DC, Fouladi-Nashta AA (2009) The effect of linolenic acid on bovine oocyte maturation and development. Biol Reprod 81:1064-1072.

Marei WF, Wathes DC, Fouladi-Nashta AA (2012) Differential effects of linoleic and alpha-linolenic fatty acids on spatial and temporal mitochondrial distribution and activity in bovine oocytes. Reprod Fertil Dev 24:679690.

McKeegan PJ, Sturmey RG (2011) The role of fatty acids . in oocyte and early embryo development. Reprod Fertil Dev 24:59-67.

Nagai T (2001) The improvement of in vitro maturation systems for bovine and porcine oocytes. Theriogenology, 55:1291-1301.

Pratt SL, Sherrer ES, Reeves DE, Stice SL (2006) Factors influencing the commercialisation of cloning in the pork industry. J Reprod Fertil Suppl 62:303-315.

Sturmey RG, Reis A, Leese HJ, McEvoy TG (2009) Role of fatty acids in energy provision during oocyte maturation and early embryo development. Reprod Domest Anim 44:50-58.

Takahashi M (2012) Oxidative stress and redox regulation on in vitro development of mammalian embryos. J Reprod Dev 58:1-9.

Wakefield SL, Lane M, Schulz SJ, Hebart ML, Thompson JG, Mitchell M (2007) Maternal supply of omega-3 polyunsaturated fatty acids alter mechanisms involved in oocyte and early embryo development in mouse. Am J Physiol Endocrinol Metab 294:E425-E434.

Wathes DC, Abayasekara DRE, Aitken RJ (2007) Polyunsaturated fatty acids in male and female reproduction. Biol Reprod 77:190-201.

Wonnacott KE, Kwong WY, Hughes J, Salter AM, Lea RG, Garnsworthy PC, Sinclair KD (2010) Dietary omega-3 and-6 polyunsaturated fatty acids affect the composition and development of sheep granulosa cells, oocytes and embryos. Reproduction 139:57-69.

Wu Y, Zhang N, Li YH, Zhao L, Yang M, Jin Y, Xu YN, Guo H (2017) Citrinin exposure affects oocytes maturation and embryo development by inducing oxidative stress-mediated apoptosis. Oncotarget 8:34525-34533. 